\title{
Exclusion Processes with Internal States
}

\author{
Tobias Reichenbach, Thomas Franosch, and Erwin Frey \\ Arnold Sommerfeld Center for Theoretical Physics (ASC) and Center for NanoScience (CeNS), Department of Physics, \\ Ludwig-Maximilians-Universität München, Theresienstrasse 37, D-80333 München, Germany
}

(Received 8 May 2006; published 2 August 2006)

\begin{abstract}
We introduce driven exclusion processes with internal states that serve as generic transport models in various contexts, ranging from molecular or vehicular traffic on parallel lanes to spintronics. The ensuing nonequilibrium steady states are controllable by boundary as well as bulk rates. A striking polarization phenomenon accompanied by domain wall motion and delocalization is discovered within a mesoscopic scaling. We quantify this observation within an analytic description providing exact phase diagrams. Our results are confirmed by stochastic simulations.
\end{abstract}

DOI: 10.1103/PhysRevLett.97.050603

Understanding the physical principles governing nonequilibrium transport in one-dimensional (1D) systems has been the subject of recent interest in both biological physics $[1,2]$ and mesoscopic quantum systems [3]. Though there are fundamental differences due to quantum coherence effects, there is a variety of common themes. One of them is the control of a nonequilibrium steady state through particle injection and extraction at the boundaries and coupling to some external field in bulk. For example, a generic spintronic scheme [3] consists of sources and drains for spin injection and extraction where the spin orientation is controlled by a tunable effective magnetic field. The analog in nonequilibrium statistical mechanics is the asymmetric simple exclusion process with open boundaries [4]. In its simplest version, particles interacting only with hard-core repulsion move unidirectionally from the left to the right boundary, which are acting as sources and drains, respectively. These particles may either correspond to molecular engines like mRNA or kinesin moving actively along molecular tracks [5] or to macromolecules driven through nanoscale pores or channels [6] by some external field. Already this simplest conceivable model for collective transport exhibits phase transitions between different nonequilibrium steady states controlled by the entrance and exit rates at the boundaries [7]. It has recently been noted that a minimal model for intracellular transport has to account for the fact that molecular motors may enter or leave the track not only at the boundaries but also in bulk $[8,9]$. Then, the bulk reservoir of particles acts as a gate, which can induce phase separation [9] in a mesoscopic limit where the residence time of the particles is of the same order as the transit time.

In this Letter, we introduce a generalization of the (totally) asymmetric simple exclusion process (TASEP) in which particles possess some discrete internal states. For illustration, we restrict the discussion to two states which are referred to as spin-up $(\uparrow)$ and spin-down $(\downarrow)$; see Fig. 1. Inspired by Pauli's exclusion principle, we allow multiple occupancy of sites only if particles are in different internal states. The resulting dynamics underlies surpris-
PACS numbers: 05.70.Ln, 05.40.- a, 05.60.- $\mathrm{k}, 72.25 .-\mathrm{b}$

ingly diverse situations. The internal states may correspond to distinct states of a molecular engine which are allowed to simultaneously occupy the same site of a molecular track and mutually inhibit each others motion. In the context of molecular [10] or vehicular [11] traffic, transport on several parallel lanes may be described by attributing internal states to vehicles (molecular motors) moving on a single lane. Even further, considering hopping transport in chains of quantum dots [12] with applied voltage, the states are directly identified with the spin of the electron. In this situation, the model specified in more detail below maps to a quasiclassical version of a nonequilibrium Ising spin chain with nearest neighbor hopping and spin flips, where particles still respect Pauli's exclusion principle but phase coherence is lost.

Motivated by this broad range of possible applications, we consider the following 1D lattice model with $L$ sites and open boundaries illustrated in Fig. 1. At the left, particles with spin-up (-down) are injected at rate $\alpha^{\uparrow}$ $\left(\alpha^{\downarrow}\right)$, respecting Pauli's exclusion principle; i.e., each site might at most be occupied by one spin-up and one spindown state. Within bulk, particles hop unidirectionally to the right at rate $r$ and may flip their spin orientation with rate $\omega$, again under the constraint of Pauli's exclusion principle. Finally, the rates $\beta^{\uparrow, \downarrow}$ control spin extraction at the right boundary. When interpreting our model as twolane traffic [11], the states correspond to lanes such that

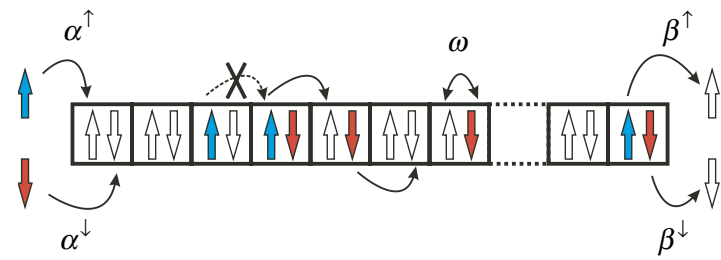

FIG. 1 (color online). Illustration of an exclusion model with two internal states. Particles in states $\uparrow(\downarrow)$ enter with rates $\alpha^{\uparrow}$ $\left(\alpha^{\downarrow}\right)$, move unidirectionally to the right within the lattice, may flip at rate $\omega$, and leave the system at rates $\beta^{\uparrow}\left(\beta^{\downarrow}\right)$, always respecting Pauli's exclusion principle. 
each site might at most be occupied once, and spin flip translates to switching between lanes.

The quantities of interest are the state-resolved density $\rho^{\uparrow, \downarrow}(x)$ and current profiles $j^{\uparrow, \downarrow}(x)$ in the nonequilibrium steady state as functions of the spatial position $x$, emerging from the interplay between external driving, coupling between the internal states (spin flips), and the exclusion principle. We identify a cooperative transition between a homogeneous state, where the density profiles of both internal states exhibit no significant spatial dependence, to a "polarized" state, where the density profile of one state changes abruptly along the track. The latter implies that the polarization $\rho^{\dagger}(x)-\rho^{\downarrow}(x)$ switches from a "low" to a "high" value at a well-defined position $x_{w}$; i.e., a domain wall forms. We find that this position can be tuned by changing the rates of spin injection (source) and extraction (drain) as well as the spin flip rate (gate). For the transition from a polarized to an unpolarized state, two scenarios emerge. Either the domain wall leaves the system continuously through the left or right boundary or it exhibits a delocalization transition. There are thus two genuinely distinct modes of switching the polarized state of the system on and off.

The system's dynamics is governed by boundary $(\alpha, \beta)$ and bulk $(r, \omega)$ rates, where from now on we fix the time scale by setting the hopping rate to unity, $r=1$. Obviously, if the spin flip rate $\omega$ is fast, i.e., comparable with the hopping rate, the spin degrees of freedom can be considered as relaxed such that the system's behavior is qualitatively the same as for TASEP without internal states [13]. However, if the typical number of spin flips that a particle performs while traversing the system becomes comparable to the entrance and exit rates, one expects the interplay between these processes to yield interesting collective effects. In order to highlight this dynamic regime, we introduce the gross spin flip rate $\Omega=L \omega$, which we choose to be of the same order as the boundary rates. A proper mesoscopic limit [9] is defined by keeping $\Omega$ fixed as the number of lattice sites $L$ tends to infinity.

Much of the system's behavior can already be inferred on the basis of symmetries and current conservation. Consider the motion of holes with a given internal state, i.e., the absence of a particle with opposite spin. These holes move from right to left and flip their spin state at the same rate $\omega$. The dynamics exhibits a particle-hole symmetry: changing the notion of particles and holes with simultaneous interchange of "left" and "right" as well as $\alpha^{\uparrow, \downarrow} \leftrightarrow \beta^{\downarrow, \uparrow}$ leaves the system's dynamics invariant. In addition, there is a spin symmetry as seen by interchanging the spin states and the injection and extraction rates, $\alpha^{\dagger} \leftrightarrow$ $\alpha^{\downarrow}$ and $\beta^{\dagger} \leftrightarrow \beta^{\downarrow}$. Let us now study the spin and particle currents passing through site $i$, denoted by $j_{i}^{\uparrow, \downarrow}$ and $J_{i}=$ $j_{i}^{\uparrow}+j_{i}^{\downarrow}$, respectively. Since particles are not allowed to leave or enter the system, except for the boundaries, the particle current $J_{i}$ is strictly conserved and thus spatially constant, $J=J_{i}$. Unlike the particle current, the spin currents are not conserved individually. Because of spin flip processes, there is a leakage current from one spin state to the other. Since spin flips typically occur on time scales comparable to the time a particle needs to traverse the system (mesoscopic limit), this leakage current is only weak, and consequently both spin currents exhibit a slowly varying spatial dependence.

Similar to TASEP, the particle current is limited either by the left or right boundary or the capacity of the bulk. The latter restricts the current to values below a maximal value of $1 / 2$. For the left boundary, the current cannot exceed $J_{\text {IN }}=\alpha^{\uparrow}\left(1-\alpha^{\uparrow}\right)+\alpha^{\downarrow}\left(1-\alpha^{\downarrow}\right)$, while the right boundary constrains it to a value not larger than $J_{\mathrm{EX}}=$ $\beta^{\uparrow}\left(1-\beta^{\uparrow}\right)+\beta^{\downarrow}\left(1-\beta^{\downarrow}\right)$. If the current is below the maximal current, it is determined by the smaller of the boundary currents: $J=\min \left(J_{\mathrm{IN}}, J_{\mathrm{EX}}\right)$ [15]. Depending on which of both cases applies, we discern two complementary regions in the five-dimensional parameter space spanned by $\alpha^{\uparrow,}$, $\beta^{\dagger, \downarrow}$, and $\Omega$. We refer to the region where the total current is given by $J_{\mathrm{IN}}$ as the injection dominated region (IN), while the case $J=J_{\mathrm{EX}}$ corresponds to the extraction dominated region (EX). Note that both regions are connected by particle-hole symmetry. Since this symmetry is discrete, we expect discontinuous phase transitions upon crossing the border from the injection to the extraction dominated region, i.e., the IN-EX boundary. At this boundary the system exhibits phase coexistence, which similar to TASEP [17] manifests itself in a delocalized domain wall between a low density (LD) and a high density (HD) state of both spin states. Thus, based on mere symmetry arguments, we conclude that across the IN-EX boundary a delocalization transitions appears. The simultaneous formation of a domain wall in the density profiles of both spin states exclusively occurs at the IN-EX boundary. This restriction also originates in the conserved particle current, as can be seen by the following argument: For the presence of a domain wall in the spin-up density profile, the spin-up current in the vicinity of the left boundary is determined by the entrance rate, $j_{i=1}^{\uparrow}=\alpha^{\uparrow}\left(1-\alpha^{\uparrow}\right)$, while at the right boundary the exit rate specifies its value to $j_{i=L}^{\dagger}=\beta^{\dagger}(1-$ $\beta^{\uparrow}$ ). If a domain wall simultaneously forms in the spindown density profile, analogous relations hold for its current. By conservation of the particle current, $J=j_{i}^{\dagger}+j_{i}^{\downarrow}$, we encounter the constraint

$$
\alpha^{\uparrow}\left(1-\alpha^{\uparrow}\right)+\alpha^{\downarrow}\left(1-\alpha^{\downarrow}\right)=\beta^{\uparrow}\left(1-\beta^{\uparrow}\right)+\beta^{\downarrow}\left(1-\beta^{\downarrow}\right),
$$

which determines the IN-EX boundary.

Even more intriguing phase behavior emerges away from the phase boundary between the injection and extraction dominated regions. In particular, we find a broad parameter regime where a localized domain wall forms in the density profile of one spin state, whereas the profile of the other spin state remains almost flat. The generic 
situation, as obtained from stochastic simulations and mean-field (MF) calculations (discussed below), is exemplified in Fig. 2 for the IN region. Both spin states enter at comparable rates, and their respective densities approach each other due to spin flips as the spins traverse the system until they reach the point $x_{w}$. There, the density of spin-up jumps to a high value (HD), while the one of spin-down remains at a low level (LD). We encounter a spontaneous polarization effect: in the vicinity of the right boundary, the densities of spin-up and -down largely differ, although they did not when entering the system. We will refer to the parameter range where this spin polarization effect occurs

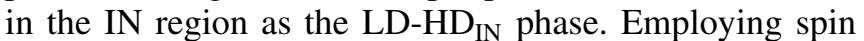
symmetry to Fig. 2 yields a domain wall appearing in the density profile of the spin-down state, while one concludes from particle-hole symmetry that there is also a corresponding $\mathrm{LD}-\mathrm{HD}_{\mathrm{EX}}$ phase in the EX region. Varying the entrance and exit rates, one can smoothly tune the domain wall position as long as the IN-EX boundary is not crossed. If $x_{w}$ passes the point $x_{w}=1$ in the situation of Fig. 2, the density of spin-up changes from the LD-HD ${ }_{\text {IN }}$ phase to the LD phase. On the other hand, $x_{w}=0$ marks the transition between the LD-HD $\mathrm{EX}_{\mathrm{EX}}$ and the HD phase. Regarding the domain wall position $x_{w}$ as an order parameter, these transitions are continuous.

The formation of a localized domain wall can be understood from the continuity of the spin currents (Fig. 2), which in turn arises from the only weak leakage current in the mesoscopic limit [9]. Say the domain wall forms in the spin-up state. Then we have to match the currents adjacent to the left $(l)$ and right $(r)$ of this wall, which in the limit of large system size are given by $j_{l / r}^{\uparrow}(x)=\rho_{l / r}^{\uparrow}(x) \times$ $\left[1-\rho_{l / r}^{\uparrow}(x)\right]$. Both currents should coincide at the position

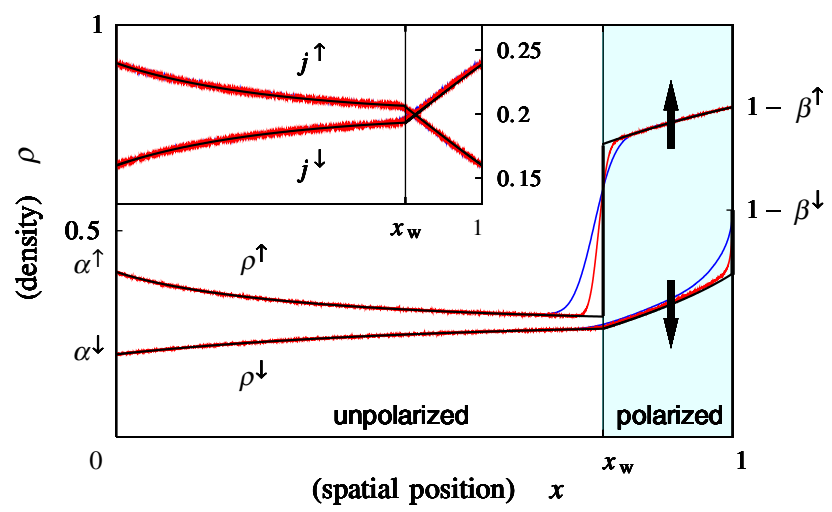

FIG. 2 (color online). The polarization phenomenon appearing in the IN region $\left(\alpha^{\uparrow}=0.4, \alpha^{\downarrow}=0.2, \beta^{\uparrow}=0.2, \beta^{\downarrow}=0.45\right.$, and $\Omega=0.5$ ). A domain wall forms in the density profile of spin-up states, while spin-down stays in a LD phase. The spin currents, shown in the inset, are both continuous. Solid lines correspond to the analytical solution, while dashed lines indicate results from stochastic simulations on lattices with $L=2000$ (blue or dark gray) and $L=10000$ (red or light gray). of the domain wall, $j_{l}^{\dagger}\left(x_{w}\right)=j_{r}^{\dagger}\left(x_{w}\right)$, while the density shows a discontinuity. Together, we arrive at the condition $\rho_{r}^{\uparrow}\left(x_{w}\right)=1-\rho_{l}^{\uparrow}\left(x_{w}\right)$ for the domain wall position $x_{w}$. The spatial dependence of the density implies that a suitable position $x_{w}$ indeed exists within a certain parameter region.

Let us now underpin the so far general discussion by a quantitative analytical description. Consider therefore the average density $\rho_{i}^{\uparrow \downarrow}$ at site $i$ for spin-up and -down states. The dynamical rules yield equations for their time evolution, which upon factorizing two-point correlations reduce to a closed set of difference equations; such MF approximations have been fruitfully applied within related contexts $[9,18,19]$. In a continuum limit, the difference equations turn into differential equations, which to leading order in the lattice constant $1 / L$ take the form

$$
\begin{aligned}
& \left(2 \rho^{\uparrow}-1\right) \partial_{x} \rho^{\uparrow}+\Omega \rho^{\downarrow}-\Omega \rho^{\uparrow}=0, \\
& \left(2 \rho^{\downarrow}-1\right) \partial_{x} \rho^{\downarrow}+\Omega \rho^{\uparrow}-\Omega \rho^{\downarrow}=0 .
\end{aligned}
$$

Together with appropriate boundary conditions arising from the entrance and exit processes, they allow a straightforward analytic solution, the details of which will be presented in a forthcoming publication [16]. We have compared the analytic solution to extensive stochastic simulations through careful finite-size scaling analysis. Upon increasing the system size $L$, the densities converge to the analytical prediction, as exemplified in Fig. 2. The observed exactness of the analytical density profiles in the limit of large systems originates, on the one hand, in the exact mean-field current-density relation in the TASEP [4]. On the other hand, the coupling of the two internal states in our model locally tends to zero when the system size $L$ is increased, such that correlations between them are washed out. The situation is somewhat analogous to TASEP combined with Langmuir kinetics [9].

The analytical approach therefore allows one to obtain the exact phase diagrams of the system. In particular, we may determine the regions where phase separation and thus the polarization phenomenon occur. In general, a large variety of different phases appears with discontinuous as well as continuous transitions between them. As anticipated by our symmetry arguments, a discontinuous transition accompanied by a domain wall delocalization occurs across the border between the injection and extraction dominated regions, which is given by Eq. (1). Continuous transitions appear inside the IN as well as the EX region, when the domain wall leaves the system at one of its boundaries: $x_{w}=0$ and $x_{w}=1$. The locations in phase space where these special domain wall positions occur, the phase boundaries, can be obtained from the analytical solution of the density profiles, as the latter reveals the position $x_{w}$; details will be presented elsewhere [16].

For illustration, we focus on the special case of equal entrance rates, $\alpha^{\uparrow}=\alpha^{\downarrow} \equiv \alpha$, which already exhibits the main features of the possible nonequilibrium steady states. In particular, when a domain wall occurs in the density 

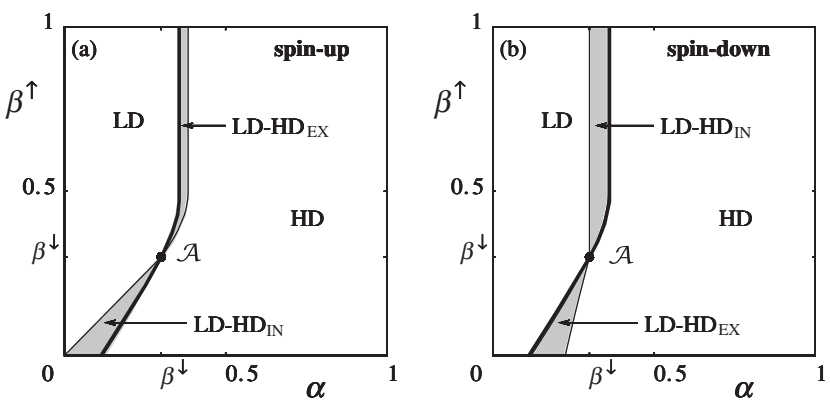

FIG. 3. Phase diagram for equal injection rates $\alpha^{\uparrow}=\alpha^{\downarrow} \equiv \alpha$. Phases for spin-up and -down are shown in panels (a) and (b), respectively, as a function of $\alpha$ and $\beta^{\uparrow}$ for fixed values $\beta^{\downarrow}=0.3$ and $\Omega=0.15$. Phase separation (shaded areas) arises in the IN as well as the EX region. The delocalization-transition line (thick line) is identical in (a) and (b). Thin lines correspond to continuous transitions.

profile of one of the spin states within the IN region, it lucidly shows the polarization phenomenon: both spins have equal densities in the vicinity of the left boundary, but strongly differ at the right one. The phase diagram for the spin-up and spin-down states, resulting from the analytical solution, is presented in Fig. 3. In the two-lane interpretation of the model, the states refer to the upper and lower lane. For both internal states the same first order line marks the transition between the IN and EX region. The polarized state (shaded area), where a domain wall appears either in the spin-up or -down state, intervenes between the LD and HD phases. There are continuous transitions from pure (LD, HD) to coexistence phases as the domain wall enters or exits the system at the boundary. The lines marking these transitions intersect the IN-EX boundary in a multicritical point $\mathcal{A}$, where all boundary rates equalize, $\alpha=\beta^{\uparrow}=\beta^{\downarrow}$.

Consider now a horizontal path through the phase diagram below the multicritical point $\mathcal{A}$. For small values of the injection rate $\alpha$ the system is in the injection dominated region, and both spin states (lanes) are in a homogeneous LD state. Then, upon crossing the phase boundary for the spin-up state, the system switches to a polarized state, similar to Fig. 2, such that a domain wall appears in the density profile of the spin-up state (upper lane), entering continuously from the right boundary; for the same parameter range the spin-down state stays in a homogeneous LD state. Approaching the IN-EX boundary this domain wall delocalizes, and upon crossing relocalizes again, but now as a domain wall in the spin-down state (lower lane). As the spin-up state has turned to a HD phase, we encounter polarization near the left boundary. Crossing the IN-EX boundary, the system thus switches its polarization from the right boundary to the left one. A further increase of the injection rate finally shifts the position of the domain wall to the left boundary such that the system remains in a HD phase for both spin states (lanes). For a path through the phase diagram above $\mathcal{A}$, similar arguments hold.

Two of the lines that mark continuous transitions are readily obtained. The transition from the $\mathrm{LD}$ to the LD-HD IN phase is determined by $x_{w}=1$; since $\rho^{\dagger}(x)=$ $\rho^{\downarrow}(x)=\alpha$ for $x<x_{w}$, it is located along the diagonal, $\alpha=$ $\beta^{\uparrow}$, for the spin-up state and parallel to the vertical axis, $\alpha=\beta^{\downarrow}$, for the spin-down state. We emphasize that these phase boundaries in the injection dominated regime do not depend on the magnitude of the gross spin flip rate $\Omega$; i.e., qualitatively tuning the system's state is possible only upon changing the injection or extraction rates. The lines corresponding to continuous transition in the EX region are more involved [16]. Its most notable feature is that the width of the polarized phase decreases with increasing spin flip rate $\Omega$, until it finally vanishes in the limit $\Omega \rightarrow \infty$.

The authors are grateful for insightful discussions with Felix von Oppen and Ulrich Schollwöck.

[1] N. Hirokawa, Science 279, 519 (1998).

[2] A. Rustom et al., Science 303, 1007 (2004).

[3] Žutić, J. Fabian, and S. D. Sarma, Rev. Mod. Phys. 76, 323 (2004).

[4] B. Derrida, Phys. Rep. 301, 65 (1998).

[5] J. Howard, Mechanics of Motor Proteins and the Cytoskeleton (Sinauer Associates, Sunderland, MA, 2001).

[6] T. Chou and D. Lohse, Phys. Rev. Lett. 82, 3552 (1999).

[7] J. Krug, Phys. Rev. Lett. 67, 1882 (1991).

[8] R. Lipowsky, S. Klumpp, and T. M. Nieuwenhuizen, Phys. Rev. Lett. 87, 108101 (2001).

[9] A. Parmeggiani, T. Franosch, and E. Frey, Phys. Rev. Lett. 90, 086601 (2003); Phys. Rev. E 70, 046101 (2004).

[10] H. Hinsch, R. Kouyos, and E. Frey, "Traffic and Granular Flow" (to be published).

[11] D. Helbing, Rev. Mod. Phys. 73, 1067 (2001).

[12] C. K. Hahn et al., Appl. Phys. Lett. 73, 2479 (1998).

[13] The physics in this limit has previously been discussed in terms of a two-lane model [14].

[14] E. Pronina and A. B. Kolomeisky, J. Phys. A 37, 9907 (2004).

[15] Strictly speaking, this holds only for rates smaller than $1 / 2$. Larger rates effectively act as $1 / 2$ [16].

[16] T. Reichenbach, T. Franosch, and E. Frey (to be published).

[17] C. MacDonald, J. Gibbs, and A. Pipkin, Biopolymers 6, 1 (1968).

[18] B. Derrida, E. Domany, and D. Mukamel, J. Stat. Phys. 69, 667 (1992).

[19] M. R. Evans et al., Phys. Rev. Lett. 74, 208 (1995). 